UDK 528.92

\title{
USE OF MERCATOR CARTOGRAPHIC REPRESENTATION FOR LANDSAT 8 IMAGERIES
}

\author{
Claudio MENEGHINI ${ }^{1}$, Claudio PARENTE ${ }^{2}$ \\ Department of Sciences and Technologies, University of Naples Parthenope, \\ Centro Direzionale - Isola C4, 80143 Naples, Italy \\ E-mails: ${ }^{1}$ claudio.meneghini@uniparthenope.it; \\ ${ }^{2}$ claudio.parente@uniparthenope.it (corresponding author) \\ Received 9 February 2017; accepted 10 May 2017
}

\begin{abstract}
Nowadays Marine Geographical Information Systems (MGIS) play an essential role in several research activities, the most part of them related to solve Geoscience problems. The nautical maps, containing most of the information used by the marine navigators, are used as cartographic base of MGIS and widely referred to Mercator projection. Remotely sensed images can be introduced in MGIS to improve the study outcomes even if they are in a different cartographic representation (generally Universal Transverse of Mercator, UTM). The adaptation of already georeferred remotely sensed images to Mercator projection requires particular care, moreover when also geodetic data are different (i.e. local datum and global datum). This paper is aimed to offer an easy-to-use work-flow that could be adopted every time remotely sensed images are to be introduced in MGIS and overlaid to nautical maps. Particularly the work addresses the implementation and evaluation of reprojection of Landsat 8 imageries, regarding both the gulfs of Naples and Salerno (Italy): a transformation from UTM WGS84 to Mercator Roma40 is applied. The result accuracy encourages the adoption of the proposed work-flow.
\end{abstract}

Keywords: Landsat-8, Marine GIS, Mercator projection, UTM, georeferencing, reprojection, datum transformation.

\section{Introduction}

Marine sciences can benefit of Geographic Information System (GIS) because of their ability to represent great volumes of multidimensional data required by these kinds of science. For this reason, Marine GIS (MGIS) is the perfect computer tool suitable to complete this task (Weintrit 2009).

In literature, many researchers have described the advantages of using GIS for marine applications. Wright (Wright 2007) highlights that MGIS technology helps to better understand the marine environment and to increase ability for measuring change in oceans and along coast.

Hamano et al. (2004) use GIS techniques to emphasize the importance of sonar information and the need to spatially map the referenced information such as the relationship between the fish schools and bottom topography. By means of GIS spatial analysis techniques, they use acoustic information from scanning sonar to determine the real features of fish schools in the water.

Ma et al. (2004) develop new GIS algorithms to both calculate the horizontal temperature gradient of fishing grid used to catch statistic and interpolate grid point temperature based on sea surface temperature (SST) isolines. The first resolves irregular several problems such as fishing grid, real distance and grid point value problem caused by existing GIS software; the latter has a wide range of applications because it can be used for interpolating grid data based on any isoline kind.

Bikonis et al. (2005) propose a prototype of safety related application of the newly developed real time and remotely accessible MGIS for water pollution monitoring and emergency management supporting. Integrating many different types of marine pollution survey data, their system processes the data acquired by acoustic sensors and supports $2 \mathrm{D}$ or $3 \mathrm{D}$ visualization and mapping of pollution aggregations. 
Claramunt et al. (2006), considering the continuous growth of maritime traffic around the world, declare that the integration of GIS with navigation systems is the direction to follow: they should overlap and continue in better unison.

The most used cartographic bases for MGIS are nautical charts that supply variegate information (i.e. navigation hazards, sea bottom relief, etc). They are produced in Mercator representation that is similar to direct cylindrical projection and derived mathematically (Guastaferro et al. 2012).

Remotely sensed imageries are very useful in MGIS for several purposes but they are mainly provided in UTM representation, which is similar to transverse cylindrical projection. To overlay satellite scenes to nautical charts, it is necessary a reprojection of those images by warping them to fit the nautical map. If this automatic reprojection is not present in GIS tools, an alternative procedure has to be found.

The goal of this paper is to define an easy-to-use work-flow to integrate remotely sensed images into MGIS overlaying them to nautical maps. For an accurate result, Ground Control Points (GCPs), uniformly distributed as a regular grid on the image, are used to georeference the scene in the new datum and projection. To evaluate the outcome image, Check Points (CPs) are considered to calculate the overall accuracy of the transformation. More precisely, Landsat 8 imageries, regarding both the gulfs of Naples and Salerno (Italy), are reprojected from UTM WGS84 to Mercator Roma40.

The paper is organized as follows. The Section 1 briefly described the cartographic representation of Mercator. In Section 2 main characteristics of Landsat 8 imageries are summarized. Section 3 illustrates the related work to georeference the nautical chart and reproject the considered satellite scene transforming its datum: the results are shown and discussed. The last Section describes the conclusion of the paper and remarks the suitable adoption of the proposed work flow for similar applications.

\section{Mercator representation}

Introduced in 1569 by Gerardus Mercator, this map projection is widely used for navigation because it represents loxodromes as straight lines (loxodrome or rhumb line is a line crossing all meridians of longitude at the same angle) (Monmonier 2010).

The Mercator cartographic representation is conformal, which means that angles and small shapes are represented correctly but the scale varies greatly away from central parts of the map, so it does not show equal areas (Croucher 2004). It is a mathematically derived projection: it is obtained by performing a regular projection of the earth model onto a cylinder and then cutting and opening the cylinder onto a plane (Canton, Sanchez 1998). More precisely, a vertical cylinder surrounds the Earth touching it at the Equator. Around it, there is a small alteration but distortions become more pronounced as the distance from Equator increases, reaching the greatest value in polar areas.

The meridians are parallel vertical and equally spaced lines while the parallels are horizontal straight parallel lines, whose distances growth with the increasement of their distances from the Equator. The Mercator equations are:

$$
\begin{gathered}
x=a\left(\lambda-\lambda_{0}\right) ; \\
y=a \ln \left[\tan \left(\frac{\pi}{4}+\frac{\varphi}{2}\right) \cdot\left(\frac{1-e \sin \varphi}{1+e \sin \varphi}\right)^{\frac{e}{2}}\right],
\end{gathered}
$$

where: $\ln$ is the neperian logarithm; $a$ is the equatorial radius; $e$ is the eccentricity of the ellipsoid.

To take advantage of the small error registered at the Equator, it is possible to modify the Mercator projection using another parallel as the contact line with the cylinder (standard parallel). In this case, the equations for the reference parallel become:

$$
\begin{gathered}
x=a\left(\lambda-\lambda_{0}\right) \cdot \frac{\cos \varphi_{1}}{\left(1-e^{2} \sin ^{2} \varphi_{1}\right)} \\
y=a \ln \left[\tan \left(\frac{\pi}{4}+\frac{\varphi}{2}\right) \cdot\left(\frac{1-e \sin \varphi}{1+e \sin \varphi}\right)^{\frac{e}{2}}\right] \frac{\cos \varphi_{1}}{\sqrt{\left(1-e^{2} \sin ^{2} \varphi_{1}\right)}},
\end{gathered}
$$

where: $\varphi_{1}$ is the latitude of the standard parallel.

\section{Landsat 8 imageries}

Landsat is an American Earth observation program born from the collaboration between the National Aeronautics and Space Administration (NASA) and the United States Geological Survey (USGS). Landsat satellites have collected, over the years, enough moderate resolution data of the entire Earth's land surface to monitor trends and to evaluate land changes (Knight, Kvaran 2014).

Landsat 8 was launched on February 11, 2013 and it represents the eighth satellite generation: its purpose 
is to provide consistency and continuity of observations making its data comparable with ones of the previous generations.

The Landsat 8 satellite carries a two-sensor payload: the Operational Land Imager (OLI) and the Thermal Infrared Sensor (TIRS) (U.S. Geological Survey 2016). The first measures in nine spectral bands in the visible and short wave infrared spectral regions while the latter measures two long-wave infrared channels as reported in Table 1.

Table 1. Landsat 8 OLI and TIRS bands

\begin{tabular}{|c|c|c|}
\hline Band name & Wavelengths $(\mu \mathrm{m})$ & \multirow{2}{*}{ Resolution } \\
\hline Coastal aerosol & $0.433-0.453$ & \\
\cline { 1 - 2 } Blue & $0.450-0.515$ & \\
\cline { 1 - 2 } Green & $0.525-0.600$ & \multirow{2}{*}{$30 \mathrm{~m}$} \\
\cline { 1 - 2 } Red & $0.630-0.680$ & \\
\hline NIR & $0.845-0.885$ & \\
\hline SWIR 1 & $1.560-1.660$ & \\
\hline SWIR 2 & $2.100-2.300$ & \\
\hline Panchromatic & $0.500-0.680$ & \multirow{2}{*}{$15 \mathrm{~m}$} \\
\hline Cirrus & $1.360-1.390$ & \multirow{2}{*}{$30 \mathrm{~m}$} \\
\hline TIRS 1 & $10.6-11.2$ & \multirow{2}{*}{$100 \mathrm{~m}$} \\
\hline TIRS 2 & $11.5-12.5$ & \\
\hline
\end{tabular}

Different spatial resolutions correspond to the bands of Panchromatic (15 meters), Visible/NIR/SWIR (30 meters) and Thermal (100 meters).

Landsat 8 orbits the Earth every 99 minutes and acquires imageries of the entire Earth every 16 days. Collecting Earth's scenes with a moderate resolution, Landsat 8 has a near-polar, sun-synchronous orbit at an altitude of $705 \mathrm{~km}$ (Allen et al. 2014). The scene size is $185 \mathrm{~km}$ cross track by $180 \mathrm{~km}$ along track.

The community of researchers worldwide uses Landsat data and there is a multitude of applications: agriculture (Cao et al. 2016), land mapping (D'Allestro, Parente 2015), temperature mapping (Cristóbal et al. 2014; Belfiore et al. 2015), pan sharpening (Belfiore et al. 2016) and change detection (Adamo et al. 2016; Bitelli et al. 2016) are only some examples.

\section{Application}

In this work, a nautical chart, regarding the Gulfs of Naples and Salerno (Italy) in scale 1:100000, is considered (Fig. 1). The considered area, referred to the Mercator representation and the Italian datum Roma40,

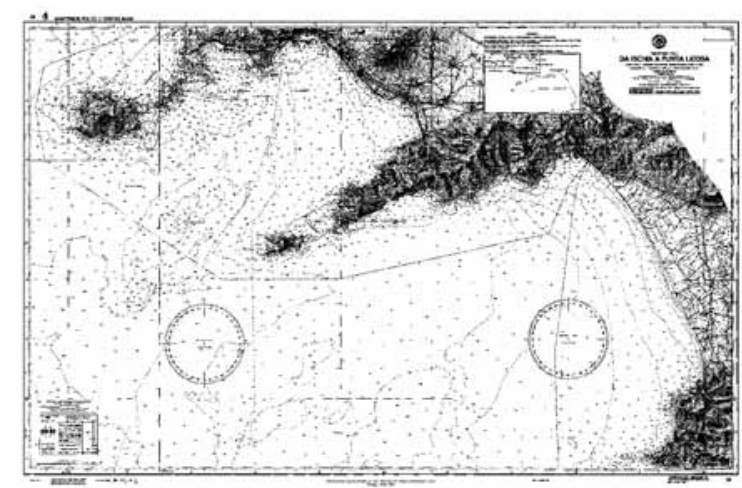

Fig. 1. The considered nautical chart

is comprised between $13^{\circ} 45^{\prime} 48^{\prime \prime} \mathrm{E}$ and $15^{\circ} 03^{\prime} 00^{\prime \prime} \mathrm{E}$ of longitude and $40^{\circ} 51^{\prime} 00^{\prime \prime} \mathrm{N}$ and $40^{\circ} 14^{\prime} 15^{\prime \prime} \mathrm{N}$ of latitude.

In this map, Mercator projection is used with another standard parallel (where length deformation is zero) instead of the Equator and located at $40^{\circ} 33^{\prime} \mathrm{N}$. To georeference the nautical map in Mercator projection, the coordinates of selected points, called Ground Control Points (GCPs), are necessary. Accurate GCPs are essential for a correct georeferencing: they must be placed homogeneously in the area of interest. In this case, they are detected as the intersections between parallels and meridians. The GCPs geographic coordinates are transformed in plane ones using the equations (2) concerning the Mercator projection adapted for the standard parallel. The formulas are included in a spreadsheet that allows Roma40 plane coordinates calculation. To georeference the nautical map in Mercator projection, a first order transformation (affine), based on polynomial functions, is applied using GIS software. Since it shifts, scales, and rotates the raster dataset, its equations are:

$$
\begin{aligned}
& X^{\prime}=a_{0}+a_{1} X+a_{2} Y ; \\
& Y^{\prime}=b_{0}+b_{1} X+b_{2} Y .
\end{aligned}
$$

For this purpose, GCPs which are identifiable in the image $(X, Y)$ must be selected accurately on the map $\left(X^{\prime}, Y^{\prime}\right)$. To calculate the six affine transformation coefficients of the polynomials $\left(a_{0}, a_{1}, a_{2}, b_{0}, b_{1}\right.$ and $b_{2}$ ), the minimum number of GCPs, whose also output coordinates are known, required to determine coefficients is three. To minimize errors introduced by georeferencing, more information in the form of extra GCPs is used (Fig. 2). Finally, 18 GCPs are considered: additional GCPs are useful to reduce the differences (residuals) between the attempted map coordinates and the resulted ones.

To determine the georeferencing accuracy, errors not only for GCPs but also for other 16 points, named 


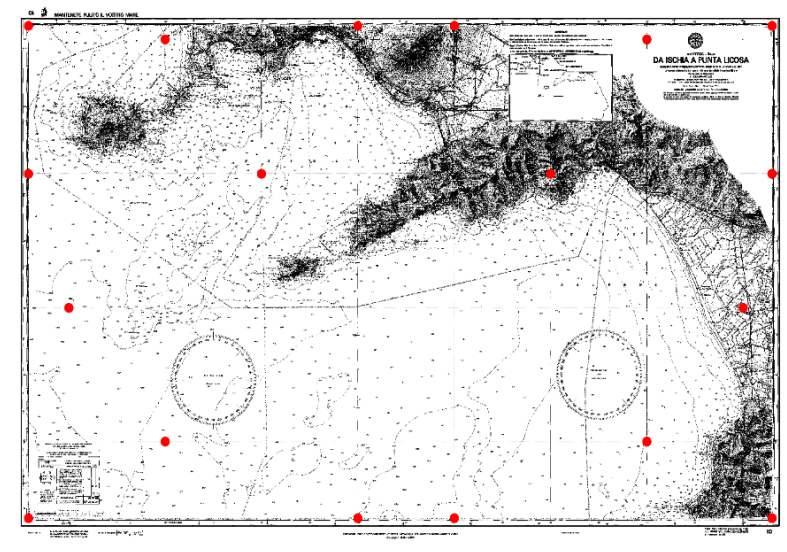

Fig. 2. GCPs on the nautical chart

Check Points (CPs), are considered (Fig. 3). Particularly several values (medium, minimum, maximum and standard deviation) of GCPs and CPs residuals in $x y$ direction are evaluated and reported in Table 2.

Table 2. Residuals (in meters) obtained for GCPs and $\mathrm{CPs}$ for nautical chart georeferencing using Polynomial Functions 1st order

\begin{tabular}{|c|c|c|c|c|}
\hline & $\begin{array}{c}\Delta x y \\
\text { medium } \\
(\mathrm{m})\end{array}$ & $\begin{array}{c}\Delta x y \\
\text { minimum } \\
(\mathrm{m})\end{array}$ & $\begin{array}{c}\Delta x y \\
\text { maximum } \\
(\mathrm{m})\end{array}$ & $\begin{array}{c}\sigma x y \\
(\mathrm{~m})\end{array}$ \\
\hline $\mathrm{GCPs}$ & 17.179 & 2.061 & 36.675 & 9.766 \\
\hline $\mathrm{CPs}$ & 22.243 & 7.645 & 36.373 & 10.030 \\
\hline
\end{tabular}

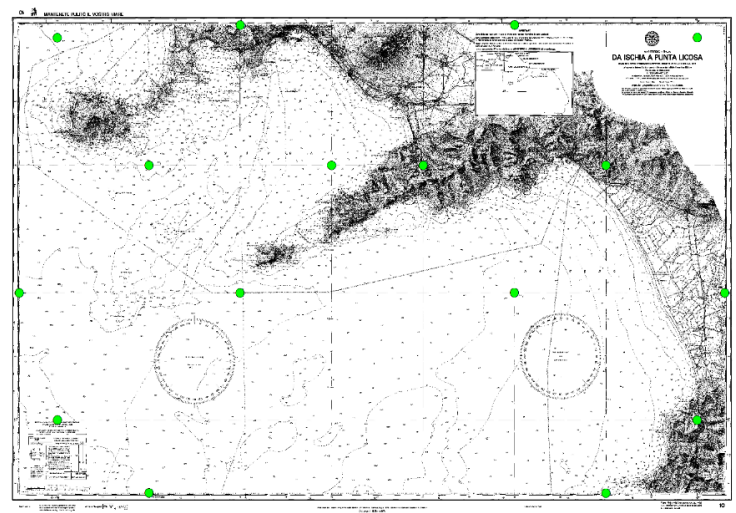

Fig. 3. CPs on the nautical chart

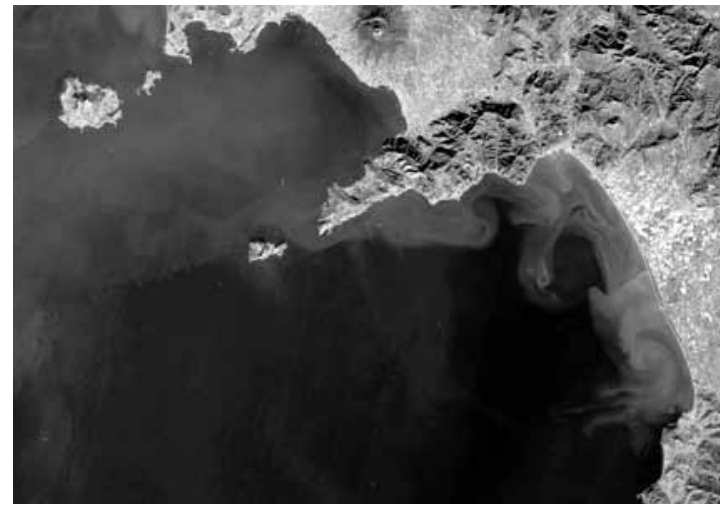

Fig. 4. The Landsat 8 OLI panchromatic image
A Landsat 8 OLI imagery of Campania Region concerning the same area of the nautical map (Gulfs of Naples and Salerno) is considered (Fig. 4). The dataset is already georeferenced in UTM/WGS84 coordinates - 33 T zone: $N_{1}=4523002.5 \mathrm{~m}, E_{1}=$ $394897.5 \mathrm{~m}, N_{2}=4454002.5 \mathrm{~m}, E_{2}=505102.5 \mathrm{~m}$.

To overlay the Landsat 8 imagery to the nautical chart, both reprojection (from UTM to Mercator) as well as datum transformation (from WGS84 to Roma40) are necessary. To accomplish these tasks the following approach is adopted. A regular point grid of 15 GCPs (Fig. 5) is chosen to cover the entire scene. The grid has an extension of $108200 \times 67000 \mathrm{~m}$ : its upper and lower coordinates are respectively $N_{1}=$ $4522000 \mathrm{~m}, E_{1}=395900 \mathrm{~m}, N_{2}=4455000 \mathrm{~m}, E_{2}=$ $504100 \mathrm{~m}$.

The GCP plane coordinates (referred to UTM/ WGS84) are transformed in geographic ones (referred to WGS84) using the corresponding equations of the Transverse of Mercator. This transformation is applied using GIS tool. To convert geographic coordinates from WGS84 to Rome40, a software developed by the Italian Minister of the Environment, named Traspunto is used. The previous spreadsheet permits to transform the geographic (Roma40) coordinates into the correspondent Mercator plane ones.

The point grid known in both plane coordinates (UTM/WGS84 and Mercator/Rome40) is used to regeoreference the satellite image and overlay it to the nautical chart. Also in this case residuals on GCPs and $16 \mathrm{CPs}$ chosen along the coast, are considered to evaluate the accuracy of the process. Particularly CPs are detected on both map and image so to obtain respectively corrected and resulting coordinates (Fig. 6).

In this case better results are achieved using transformation of second order rather than first order. Transformation of second order, in addition to the previous (affine) transformation, allows for torsion and

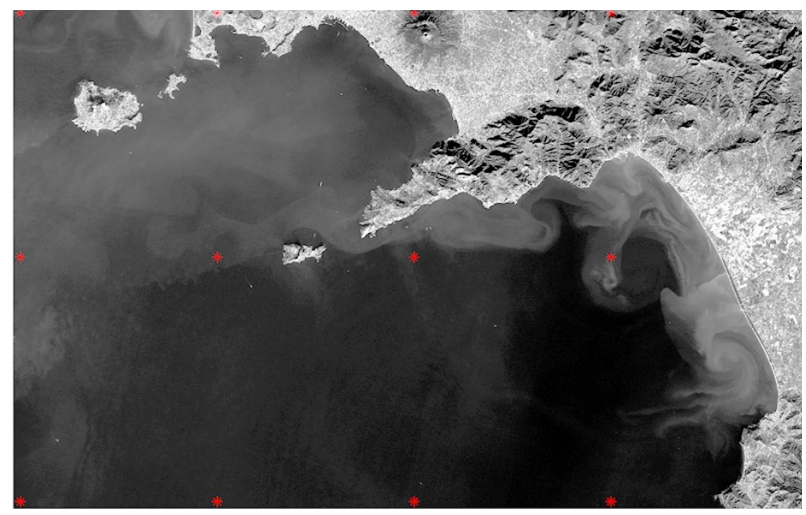

Fig. 5. The regular grid of GCPs 


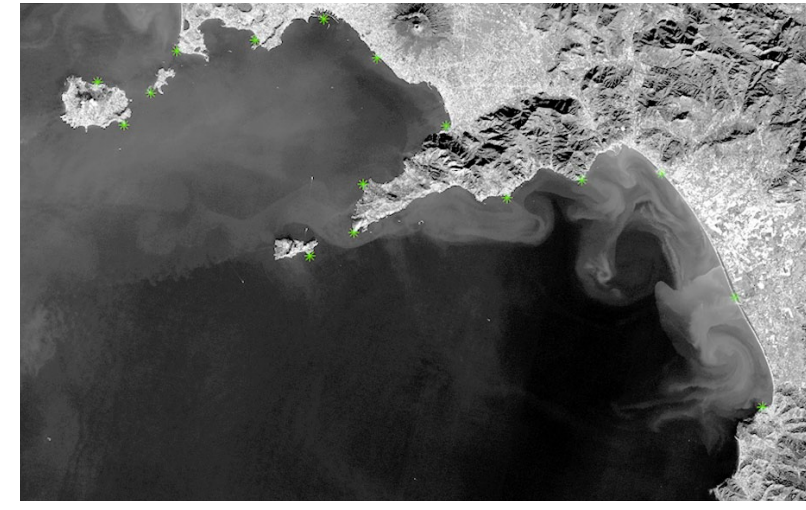

Fig. 6. The CPs homogenously distributed along the coast

convexity in both axes. The equations are:

$$
\begin{aligned}
& X^{\prime}=a_{0}+a_{1} X+a_{2} Y+a_{3} X Y+a_{4} X^{2}+a_{5} Y^{2} \\
& Y^{\prime}=b_{0}+b_{1} X+b_{2} Y+b_{3} X Y+b_{4} X^{2}+b_{5} Y^{2},
\end{aligned}
$$

where: $X^{\prime}$ and $Y^{\prime}$ are respectively the horizontal and vertical values in cartographic space; $X$ and $Y$ are respectively column and row counts in image space.

Residuals for second order transformation are shown in Table 3.

Table 3. Residuals (in meters) obtained for GCPs and CPs for Landsat 8 scene re-projection (with datum transformation) using Polynomial Functions 2nd order

\begin{tabular}{|c|c|c|c|c|}
\hline & $\begin{array}{c}\Delta x y \\
\text { medium } \\
(\mathrm{m})\end{array}$ & $\begin{array}{c}\Delta x y \\
\text { minimum } \\
(\mathrm{m})\end{array}$ & $\begin{array}{c}\Delta x y \\
\text { maximum } \\
(\mathrm{m})\end{array}$ & $\begin{array}{c}\sigma x y \\
(\mathrm{~m})\end{array}$ \\
\hline GCPs & 1.134 & 0.056 & 1.631 & 1.266 \\
\hline CPs & 5.289 & 0.415 & 10.235 & 5.790 \\
\hline
\end{tabular}

The good overlapping between the Landsat 8 image and nautical chart of the same zone in Mercator representation confirms the good accuracy of results (Fig. 7).

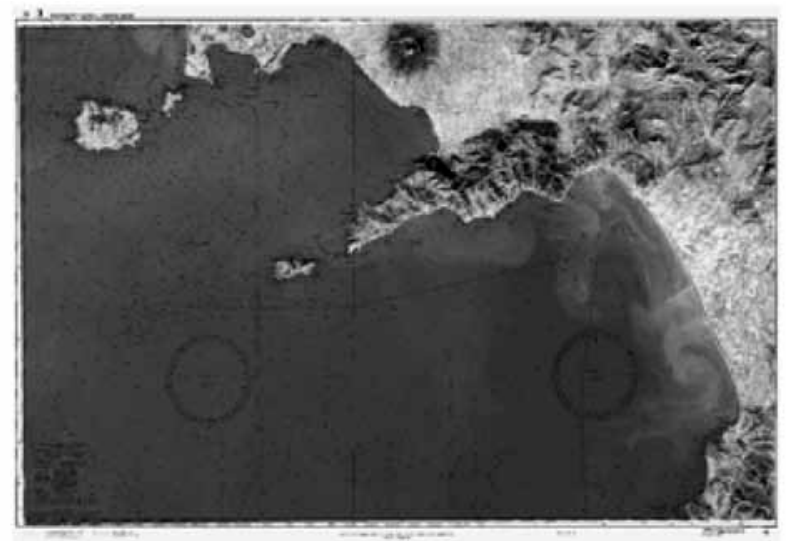

Fig. 7. The overlapping between the nautical chart and the Landsat 8 image

\section{Conclusions}

Nowadays re-projection and datum transformation are fundamental tasks to use both a nautical chart and a satellite imagery in MGIS applications. In this work, both operations are performed for Landsat 8 image in UTM WGS 84 to overlay it to nautical chart in Mercator/Roma40. GCPs, regularly spaced and known in both UTM/WGS84 and Mercator/Roma 40 plane coordinates, are fundamental to have a good performance. UTM/WGS84 coordinates are transformed in WGS84 geographical ones using GIS software. Traspunto software is used to transform WGS84 geographical coordinates in Roma40. A spreadsheet is implemented to transform geographical Roma40 coordinates in Mercator Roma40 plane ones. GIS software permits to re-georeference the Landsat scene using GCPs and Polynomial Functions 2nd order. CPs, which coordinates are detected in both systems (UTM/WGS84 and Mercator/Roma40 plane coordinates) confirms the accuracy of the final products.

\section{Acknowledgements}

This research was part of the "New Geodetic Reference System and GNSS data quality" project supported by the University of Naples "Parthenope". We would like to thank Prof. Raffaele Santamaria, the Director of the Department of Sciences and Technologies, for his scientific support of our research activities.

\section{Disclosure statement}

Authors do not have any competing financial, professional or personal interests from other parties.

\section{References}

Adamo, M.; Blonda, P.; Lucas, R.; Tarantino, C. 2016. Detection of changes in semi-natural grasslands by cross correlation analysis with WorldView-2 images and new Landsat 8 data, Remote Sensing of Environment 175: 65-72. https://doi.org/10.1016/j.rse.2015.12.031

Allen, R. G.; Anderson, M. C.; Loveland, T. R.; Roy, D. P.; Scambos, T. A.; Woodcock, C. E.; Wulder, M. A. 2014. Landsat-8: Science and product vision for terrestrial global change research, Remote Sensing of Environment 145: 154-172. https://doi.org/10.1016/j.rse.2014.02.001

Belfiore, O. R.; D’Allestro, P.; Meneghini, C.; Parente, C. 2016. QGIS use for IHS PAN-Sharpening Application to Landsat 8 OLI images, Advanced Science Letters 22(10): 2773-2776.

Belfiore, O. R.; Giannini, M. B.; Parente, C.; Santamaria, R. 2015. Land surface temperature from Landsat 5 TM images: comparison of different methods using airborne thermal data, Journal of Engineering Science and Technology Review 8(3): 83-90. 
Bikonis, K.; Demkowicz, J.; Lubniewski, Z.; Moszynski, M.; Partyka, A.; Stepnowski, A. 2005. Nearly real-time, safety-related, remotely accessible marine GIS, in IEEE Oceans 2005 Europe Conference, 20-23 June 2005, Brest, France.

Bitelli, G.; Karnieli, A.; Zanchetta, A. 2016. Monitoring desertification by remote sensing using the Tasselled Cap transform for long-term change detection, Natural Hazards, 83(223): 223-237.

Canton, M. P.; Sanchez, J. 1998. Space image processing. 1st ed. CRC Press, 186-187.

Cao, W.; Cheng, T.; Tian, Y.; Xu, X.; Yao, X.; Zhu, Y. 2016. Mapping rice planting area from Landsat 8 imagery using one-class support vector machine, in The Fifth International Conference on Agro-Geoinformatics, 18-20 July 2016, Tianjin, China.

Claramunt, C.; Petit, M.; Ray, C. 2006. A contextual approach for the development of GIS: application to maritime navigation, in International Symposium on Web and Wireless Geographical Information Systems, 4-5 December 2006, Hong Kong, China.

Cristóbal, J.; Jiménez-Muñoz, J. C.; Mattar, C.; Skoković, D.; Sobrino, J. A. 2014. Land surface temperature retrieval methods from Landsat-8 thermal infrared sensor data, Geoscience and Remote Sensing Letters 11(10): 1840-1843. https://doi.org/10.1109/LGRS.2014.2312032

Croucher, P. 2004. JAR professional pilot studies. 1st ed. Electrocution Technical Publishers, 3-4.

D'Allestro, P.; Parente, C. 2015. GIS application for NDVI calculation using Landsat 8 OLI images, International Journal of Applied Engineering Research 10(21): 42099-42102.

Guastaferro, F.; Maglione, P.; Parente, C. 2012. Rectification of spot 5 satellite imagery for marine geographic information systems, in Advanced Research in Scientific Areas Virtual International Conference, 3-7 December 2012.

Hamano, A.; Nakamura, T.; Tanoue, H.; Umetani, Y. 2004. Application of marine GIS using information from sonar for coastal fisheries, in OCEANS'04 - MTTS/IEEE TECHNOOCEAN'04, 9-12 November 2004, New York, USA.

Knight, E. J.; Kvaran, G. 2014. Landsat-8 operational land imager design, characterization and performance, Remote Sensing 6(11): 10286-10305. https://doi.org/10.3390/rs61110286

Ma, W.; Rong, K.; Shao, Q.; You, Z. 2004. Error analysis and a new arithmetic study on computing horizontal temperature gradient, in 2004 IEEE International Geoscience and Remote Sensing Symposium Proceedings, 20-24 September 2004, Anchorage, USA.

Monmonier, M. 2010. Rhumb lines and map wars: a social history of the mercator projection. 1st ed. University of Chicago Press, 1-7.

U.S. Geological Survey. 2016. Landsat8 data users handbook [online], [cited 29 January 2017]. Available from Internet: https://landsat.usgs.gov/documents/Landsat8DataUsersHandbook.pdf

Weintrit, A. 2009. The Electronic Chart Display and Information System (ECDIS): an operational handbook. 1st ed. CRC Press.

Wright, D. J. 2007. Arc marine: GIS for a blue planet. 1st ed. ESRI Press, 1-3.

Claudio MENEGHINI. Graduated in Applied Informatics (curriculum: Geomatica), he has discussed a Thesis on Application of Change detection techniques to High resolution images using free and open source GIS software. At present, he is fellowship at the Department of Sciences and Technologies, University of Naples "Parthenope". His research activity interests Remote sensing, Image processing, Very High Resolution Satellite images, Pan-sharpening Methods, Shadow detection and removal.

Claudio PARENTE. He is Associate Professor at the Department of Sciences and Technologies, University of Naples "Parthenope" for Scientific and Disciplinary Group ICAR/06 Geomatics (Topography and Cartography). Graduated with full marks in Civil Engineering (Territorial Planning) at University of Naples "Federico II", he obtained Master in Sciences and Engineering of Sea and PhD in Geodetic and Topographic Sciences at Naval Institute of Naples. He has participated to research projects financed by MURST, MIUR, UE and Campania Region, taking care himself of GIS, Cartography and Remote sensing. He is member of the College of Teachers of Research Doctorate in Geomatica, Navigation and Geodesy. He is author or co-author of more than 90 papers published in scientific journals or proceedings of National or International Conferences. 